\title{
Combined phosphodiesterase inhibitors in end-stage heart failure: A case presentation
}

\author{
Javier Reyna ${ }^{1}$, Julio G. Peguero ${ }^{2}$, Esteban Escolar ${ }^{1}$, Orlando Santana ${ }^{1 *}$, Gervasio A. Lamas ${ }^{1}$ \\ ${ }^{1}$ Division of Cardiology at Mount Sinai Medical Center, Columbia University, Miami Beach, USA \\ ${ }^{2}$ The Division of Internal Medicine at Mount Sinai Medical Center, Columbia University, Miami Beach, USA \\ Email: "osantana@msmc.com
}

Received 17 March 2013; revised 18 April 2013; accepted 22 May 2013

Copyright @ 2013 Javier Reyna et al. This is an open access article distributed under the Creative Commons Attribution License, which permits unrestricted use, distribution, and reproduction in any medium, provided the original work is properly cited.

\begin{abstract}
Heart failure (HF) is the leading cause of morbidity and mortality and is evolving to epidemic proportions. Despite pharmacologic advances and device interventions, HF remains a progressive disease. The phosphodiesterase inhibitors-3 (PDE3I), and more recently, the phosphodiesterase inhibitors-5 (PDE5I) have been used as part of the treatment in certain patients, however, such combination has not been studied or reported before.
\end{abstract}

Keywords: Heart Failure; Phosphodiesterase Inhibitors

\section{INTRODUCTION}

Heart failure (HF) is the leading cause of morbidity and mortality. Although, much progress has been made in the pharmacotherapy, and in the development of mechanical devices for HF, it still portends a poor prognosis. The phosphodiesterase inhibitors-3 (PDE3I), mainly milrinone has been a mainstay in the therapy of HF. Recently the phosphodiesterase inhibitors-5 (PDE5I) have been reported as a potential treatment in certain patients, however the combination of the two drugs has not been studied, or previously reported. We report such a case.

\section{CASE PRESENTATION}

A 66-year-old man presented to our institution in NYHA heart failure class IV. He had a 10-year history of a non-ischemic cardiomyopathy with ejection fraction of $20 \%$, severe functional mitral insufficiency, atrial fibrillation, diabetes mellitus, chronic obstructive pulmonary disease, and chronic kidney disease with a glomerular filtration rate of $23 \mathrm{ml} / \mathrm{min}$. An implantable cardio-defibrillator was placed 2 years prior to admission. Maximal

"Corresponding author. medical therapy improved his fluid overload, but marginally improved his functional status. His defibrillator was upgraded to biventricular pacing without evident clinical benefit.

After tailored therapy guided with an indwelling SwanGanz catheter, the hemodynamics were: mean right atrial pressure of $9 \mathrm{mmHg}$, pulmonary artery pressure 45/26 mmHg with a mean of $32 \mathrm{mmHg}$, pulmonary capillary wedge pressure of $19 \mathrm{mmHg}$, and a systemic blood pressure of 100/72 mmHg. The mixed venous oxygen saturation was $56.5 \%$. The cardiac output and cardiac index were $2.4 \mathrm{lpm} / \mathrm{m}^{2}$ and $1.2 \mathrm{lpm} / \mathrm{m}^{2}$, respectively. After 30 minutes of milrinone infusion at $0.5 \mathrm{mcg} / \mathrm{kg} / \mathrm{min}$, the pulmonary capillary wedge pressure was $9 \mathrm{mmHg}$, the cardiac output and cardiac index were $3.6 \mathrm{lpm} / \mathrm{m}^{2}$ and $1.7 \mathrm{lpm} / \mathrm{m}^{2}$, respectively, and the mixed venous oxygen saturation was $62.5 \%$. The milrinone infusion was continued as an outpatient. He was rejected for cardiac transplant at several centers in the country, but accepted for destination biventricular assist device. The patient refused the device, gave up on life, and returned home. He continued declining clinically, despite maximal medical therapy including milrinone infusion (INTERMAC profile 2 - 3).

Based on positive preliminary clinical evidence of PDE5 inhibition in congestive HF and the lack of alternatives, the decision was made to add tadalafil to his regimen. The drug was titrated from $5 \mathrm{mg}$ to $20 \mathrm{mg}$ daily over a period of 4 weeks. He noticed a clinical improvement within 2 weeks of treatment. After 3 months, he was able to walk more than two blocks. He stated that he was "feeling better than at any other time in the past 3 years". His renal function improved to a glomerular filtration rate of $40 \mathrm{ml} / \mathrm{min}$. Four months after starting the therapy, he started a new business, and became sexually active. Two months after this, his physicians witnessed a brisk climb of 2 flights of stairs during a birthday cele- 
bration. His most recent echocardiogram showed no significant changes. He has not been hospitalized since the tadalafil was started. On his last office visit, 8 month after starting the therapy, he was euvolemic and symptom-free.

\section{DISCUSSION}

A PDE3 inhibitor, such as milrinone, acts by decreasing the rate of cyclic adenosine monophosphate degradation and enhancing calcium influx into the cell, which in turn increases contractility. The PDE5 inhibitors are widely used for erectile dysfunction, however the enzyme is found in the pulmonary vasculature and is up-regulated in patients with heart failure [1-4]. Preclinical studies suggest a favorable myocardial effect of PDE5 inhibitors by blocking adrenergic, hypertrophic and pro-apoptotic signaling, thereby supporting their use in HF $[1,4]$. The use of PDE5 inhibitors in HF is off-label. However, small studies have shown that they increase oxygen uptake, increase cardiac index, reduce pulmonary systemic vascular resistance, reduce aortic stiffness and improve exercise time [5]. There is preliminary evidence that suggests some benefit in systolic HF $[2,6]$, especially in patients with concomitant secondary pulmonary hypertension and right ventricular failure [7-9].

The mechanism of the benefit of the PDE5 inhibitors in HF is not clear. The vasodilator effect, especially in the pulmonary vasculature, and the improvement of endothelial function due to an increase on nitric oxide, and in general the enhanced ventriculo-vascular coupling, may explain some of the benefits [2-4,7]. Another interesting hypothesis is that due to the poor expression of PDE5 in the heart, the PDE5 inhibitors will cross-react with PDE3 receptors promoting increased intracellular calcium and better myocardial contraction [1].

The concomitant use of PDE3 and PDE5 inhibitors has, to our knowledge, never been reported. The dramatic clinical improvement seen in our patient, suggest that PDE5 inhibition needs to be investigated as a treatment option, and may have an added effect when combined with PDE3 inhibition, in patients with severe symptomatic biventricular heart failure and group II pulmonary hypertension who do not respond to optimal standard therapy. However, this observation raises more questions than it answers, since this could be the result of multiple factors on this particular patient.

\section{REFERENCES}

[1] Nagendran, J., Archer, S.L., Soliman, D., et al. (2007) Phosphodiesterase type 5 is highly expressed in the hypertrophied human right ventricle, and acute inhibition of phosphodiesterase type 5 improves contractility. Circulation, 116, 238-248.

doi:10.1161/CIRCULATIONAHA.106.655266

[2] Guazzi, M. (2008) Clinical use of phosphodiesterase-5 inhibitors in chronic heart failure. Circulation Heart Failure, 1, 272-280.

doi:10.1161/CIRCHEARTFAILURE.108.802116

[3] Schwartz, B.G., Levine, L.A., Comstock, G., et al. (2012) Cardiac uses of phosphodiesterase-5 inhibitors. Journal of the American College of Cardiology, 59, 9-15. doi:10.1016/j.jacc.2011.07.051

[4] Kanwar, M., Agarwal, R., Barnes, M., et al. (2012) Role of phosphodiesterase-5 inhibitors in heart failure: Emerging data and concepts. Current Heart Failure Reports, 10, 26-35. doi:10.1007/s11897-012-0121-9

[5] Lewis, G.D., Shah, R., Shahzad, K., et al. (2007) Sildenafil improves exercise capacity and quality of life in patients with systolic heart failure and secondary pulmonary hypertension. Circulation, 116, 1555-1562. doi:10.1161/CIRCULATIONAHA.107.716373

[6] Guazzi, M., Samaja, M., Arena, R., et al. (2007) Longterm use of sildenafil in the therapeutic management of heart failure. Journal of the American College of Cardiology, 50, 2136-2144. doi:10.1016/j.jacc.2007.07.078

[7] Reichenbach, A., Al-Hiti, H., Malek, I., et al. (2012) The effects of phosphodiesterase 5 inhibition on hemodynamics, functional status and survival in advanced heart failure and pulmonary hypertension. International Journal of Cardiology, Published Ahead of Print. doi:10.1016/j.ijcard.2012.09.074

[8] Kalogeropoulos, A.P., Vega, J.D., Smith, A.L., et al. (2011) Pulmonary hypertension and right ventricular function in advanced heart failure. Congestive Heart Failure, 17, 189-198. doi:10.1111/j.1751-7133.2011.00234.x

[9] Di Salvo, T.G. (2012) Pulmonary hypertension and right ventricular failure in left ventricular systolic dysfunction. Current Opinion in Cardiology, 27, 262-272. doi:10.1097/HCO.0b013e3283522098 